\title{
Clinical Practice Examples of Dachaihu Decoction
}

\author{
Hongmei Zhu \\ Department of Chinese Medicine, Medical School, Xiamen University, Xiamen, China \\ Email: z5913778@126.com
}

Received February 27, 2013; revised April 26, 2013; accepted May 17, 2013

\begin{abstract}
Copyright (c) 2013 Hongmei Zhu. This is an open access article distributed under the Creative Commons Attribution License, which permits unrestricted use, distribution, and reproduction in any medium, provided the original work is properly cited.
\end{abstract}

\begin{abstract}
This paper mainly describes the experiences of clinical application of Dachaihu Decoction in treating chronic pelvic infection, coronary heart disease, insomnia, and hepatolithiasis.
\end{abstract}

Keywords: Dachaihu Decoction; Coronary Heart Disease; Insomnia; Chronic Pelvic Infection; Hepatolithiasis

\section{Introduction}

Dachaihu Decoction, originating from Treatise on Febrile Diseases, is a commonly used formula in clinic. Modifying from Xiaochaihu Decoction by excluding Radix et Rhizoma Glycyrrhizae, Radix et Rhizoma Ginseng and including Radix et Rhizoma Rhei, Fructus Aurantii Immaturus, and Radix Paeoniae Alba, it aims at harmonizing Shaoyang and relieving excess Fu syndrome by promoting defecation. It is used to treat syndrome of pathogenic evils in Shaoyang combining interior excess in Yangming by Zhang Zhongjing. In clinical practice, the formula has been flexibly modified to treat varied miscellaneous diseases with significant effects by focusing on its pathogenesis, namely, Shaoyang disorder with $\mathrm{Fu}$ qi obstruction. Now the writer would like to give four detailed examples as follows.

\section{Coronary Heart Disease}

Mr. Bai, male, $61 \mathrm{yr}$, who has coronary heart disease for over five years with chest distress in anterior thoracic region at intervals, initially came to my clinic for treatment on July 8th, 2010. He used to take Compound Danshen Tablet and Diao Xinxuekang Capsule to relieve symptoms. With the months getting hotter, Bai noticed the aggravated symptoms such as frequent occurrence of chest pain, together with shortness in breath, fatigue, dry mouth and bitter taste. The patient added that he has abdominal distention in the afternoon in recent two to three years, especially after the nap, with difficulty in defecation. Last year, he had three severe abdominal distentions with no defection and was diagnosed as intestinal obstruction. The condition got relieved then after appropriate treatment, but still occurred sometimes. At that time, the patient showed dark red tongue, with thin yellow coating and dark sublingual venae, thready and taut pulse for left hand, taut, slippery and rapid for right hand. He was diagnosed as chest obstruction with stagnated heat obstructing chest, combining Yangming excess syndrome. The method of regulating Shaoyang, removing blood stasis to unblock the channels, eliminating excess fu syndrome was selected and the combined Dachaihu Decoction with Guizhi Fuling Tablet was modified as follows: Radix Bupleuri 12 g, Radix Scutellariae 10 g, Rhizoma Pinelliae Praeparata 10 g, Radix Codonopsis 10 g, Fructus Aurantii Immaturus 10 g, Rhizoma Zingiberis Recens 3 slices, Fructus Jujubae 10 g, Radix Paeoniae Rubra 10 g, Radix Paeoniae Alba 10 g, Radix et Rhizoma Rhei 6 g, Ramulus Cinnamomi 6 g, Poria 15 g, Cortex Moutan 10 g, Radix et Rhizoma Salviae Miltiorrhizae $15 \mathrm{~g}$, Semen Persicae $10 \mathrm{~g}$, Semen Armeniacae Amarum 10 g, and Fructus Trichosanthis 20 g. One week later, his chest pain disappeared, chest distress relieved greatly, abdominal distention occurred only occasionally, and bowel movement better regulated. Then, the patient was suggested to take the decoction for another one week, with Radix et Rhizoma Rhei $6 \mathrm{~g}$ in the previous formula increased to $9 \mathrm{~g}$, and Fructus Cannabis $18 \mathrm{~g}$ included. Later, the patient telephoned to inform us that everything was good.

Comment: Coronary heart disease pertains to Chest obstruction in Chinese medicine, in which the approach of invigorating qi, accelerating blood movement and removing phlegm is frequently used. In this case, the patient only showed deficiency syndrome manifested by symptoms such as shortness in breath and fatigue, and excess syndrome in pulses, just as Synopsis of Golden Chamber-Chest obstruction, heart pain and shortness in 
breath states: For patient with no obvious aversion to cold or hot, if he shows shortness in breath, he has an excess syndrome [1]. This chest obstruction is an excess syndrome caused by phlegm-heat obstructing chest, where Shaoyang meridian covers. For patient with problems in Shaoyang meridian, he usually has symptoms such as bitter taste, dry throat and taut pulse [2]. It is beyond doubt that in this case, the disease was resulted from Shayang meridian, combining symptoms such as abdominal distention and constipation. Thus, Dachaihu Decoction was used to clear away stagnated heat in Shaoyang, regulating Shaoyang meridian, and eliminating excess $\mathrm{Fu}$ syndrome in Yangming. Together with Guizhi Fuling Tablet, which specializes in accelerating blood circulation and removing stasis and phlegm, the formula meets the syndrome and focuses on regulating both qi and blood, phlegm and blood stasis with significant effects.

\section{Insomnia}

Ms Lin, female, 46yrs, first turned to me for help on May 11th, 2010. Since diagnosed as insomnia ten years ago, she has tried varied Western and Chinese medicine, only to find herself severely disappointed. According to Lin, she always have chest suppression, and feel upset, palpitation, sense of frightening and difficulty in falling sleep every night, which commonly lead to sleepless all night. Meanwhile, Lin has dry mouth and bitter taste, discomfort in stomach, constipation, dark red tongue with yellow greasy coating, deep, thready and slippery pulse. She was diagnosed as insomnia with heart qi deficiency, phlegm-heat disturbance internally combining heart and gallbladder inquietude in Chinese medicine. Thereby the approach of tonifying heart qi, clearing heat in heart and gallbladder, removing phlegm-heat and calming down the spirit was prescribed and Chaihu Jia Longgu Muli Decoction was modified as follows: Radix Bupleuri 12 g, Radix Scutellariae 10 g, Rhizoma Pinelliae Praeparata 10 g, Radix Codonopsis 10 g, Ramulus Cinnamomi 6 g, Radix et Rhizoma Rhei Praeparata 5 g, Fossilia Ossis Mastodi 30 g, Concha Ostreae 30 g, Sclerotium Poriae Pararadicis $20 \mathrm{~g}$, Rhizoma Zingiberis 3 slices, Fructus Jujubae 10 g, Cortex Albiziae 20 g, Caulis Polygoni Multiflori 20 g, Radix et Rhizoma Salviae Miltiorrhizae 20 g, Radix Curcumae 10 g, and Semen Ziziphi Spinosae 20 g. Five days later, the patient told us that she can now sleep for 4 hours every night with her chest suppression reduced, upset and palpitation relieved. In consideration of her stubborn constipation, Radix Codonopsis $10 \mathrm{~g}$ and Ramulus Cinnamomi $5 \mathrm{~g}$ in the previous formula were excluded, Radix et Rhizoma Rhei Praeparata 5 g replaced by Radix et Rhizoma Rhei 9 g, but Fructus Aurantii Immaturus $10 \mathrm{~g}$ and Radix Paeoniae Alba $15 \mathrm{~g}$ were included. Two days later, her bowel movement was promoted a little, chest suppression eradicated with more than five hours' sleep every night. Later, the decoction was suspended due to the hysteromyoma surgery, which brought back the chest suppression. At present, the patient shows red tongue with yellow coating at the root, thready and slippery pulse, no dry mouth or bitter taste regular movement by taking more fruits and vegetable. She is suggested to take the formula ten more days to consolidate effects.

Comment: In this case, the insomnia started after delivery ten years ago, it is not only long in course but also leads to intermingled deficiency and excess syndrome. Heart qi deficiency, together with phlegm-heat disturbing heart and gallbladder, brought about all the symptoms mentioned above. At the beginning, its syndrome fit into Chaihu Jia Longgu Muli Decoction, which resulted in obvious effects. Since heart qi was promoted and phlegmheat was eliminated, symptoms such as palpitation and upset were relieved gradually, yet constipation existed as before. Hence, in her second visit, Dachaihu Decoction was applied mainly, combining drugs for calming down spirit, so as to promote bowel movement, clear away the internal phlegm-heat and clam down heart and gallbladder, thus freeing the patient from her stub-born insomnia.

\section{Chronic Pelvic Infection}

Ms Lin, female, 31 yr who initially called on my clinic on April 17th, 2008, has already had pain and sagging distention in the lower abdomen for over three years with increased pain before or after the menstruation. Her menstruation was described as profuse but dark with blood clots. The patient had chronic pelvic infection and left oviduct obstruction in accordance with Western medicine. Other symptoms were stated as follows: dry mouth, bitter taste, sore waist, breast distending pain before menstruation, disturbance of frequent dreams, irritable feeling, constipation (once a week), dark and purple tongue with slight yellow but thick greasy coating, taut, thready but rapid pulse. Lin was diagnosed as abdominal pain with stagnant heat in Chong and Ren Channels, combining excess Fu syndrome in Yangming by Chinese medicine. In consequence, the approach of clearing away the heat located in Shaoyang, dissipating blood stasis and unblocking channels, relieving excess Fu syndrome though promoting defecation was determined and the combined Dachaihu Decoction with Guizhi Fuling Tablet was modified as follows: Radix Bupleuri 15 g, Radix Paeoniae Rubra 12 g, Radix Paeoniae Alba 12 g, Rhizoma Pinelliae Praeparata 10 g, Radix et Rhizoma Rhei Praeparata 9 g, Ramulus Cinnamomi 9 g, Cortex Moutan 10 g, Radix et Rhizoma Salviae Miltiorrhizae 20 g, Poria 15 g, Semen Persicae 10 g, Fructus Aurantii Immaturus 10 g, Radix Scutellariae 10 g, Radix et Rhizoma Glycyrrhizae Praeparata cum Melle 6 g, Caulis Sargentodoxae 20 g, Radix Dipsaci 15 g, Fructus Toosendan 10 g, Rhi- 
zoma Corydalis $10 \mathrm{~g}$, Concha Ostreae $30 \mathrm{~g}$, and Semen Ziziphi Spinosae $15 \mathrm{~g}$ and prescribed to the patient. Five days later, the abdominal pain was greatly relieved with smoothing bowel movement and after another one month, the abdominal pain was eliminated completely with regular bowel movement and menstruation. Later, with combined Guizhi Fuling Tablet and Dan Zhi Xiaoyang Tablet as consolidation therapy for two more months, the patient obtained a negative result at left oviduct B-ultrasonic examination.

Comment: the Liver Meridian of Foot-Jueyi travels around the reproductive organs and reaches to lower abdomen [3]. Abdominal pain in gynaecology usually occurs at lower abdomen, therefore, it can be treated from the liver meridian: for deficiency syndrome, one can use Danggui Shaoyao Decoction to nourish blood and regulate liver, Dachaihui Decoction for excess syndrome to promote qi movement, expel heat and eliminate the excess. Moreover, since gynecological diseases often go deep to the blood phrase and Dachaihu Decoction mainly focuses on regulating qi, one should add Guizhi Fuling Tablet, which specializes in accelerating blood movement, removing stasis and promoting urination in order to strengthen its effects. In this case, with typical Shangyang syndrome, Yangming syndrome as well as qi stagnation and blood stasis syndrome, it is very effective to use combined Dachaihu Decoction with Guizhi Fuling Tablet.

\section{Hepatolithiasis}

Ms Zhang, female, 69 yrs, a retired doctor who has suffered from recurrent epigastric pain over ten years with more frequent occurrence in recent two years, paid her first visit here on May 10th, 2008. She used to take gastric motor drugs, spasm relaxants, or antibiotics to relieve pain; however, during the last three months recurrence, all the medicine did not help a little. Barium meal indicated chronic superficial gastritis and B-ultrasonic examination results showed negative results (She had cholecystectomy twenty years ago due to cholelithiasis). The patient described her symptoms as follows: continuous distension or pain in the stomach, reluctance to eat, slight nausea, bitterness in the mouth, annoyed and perplexed feeling, scanty, soft and impeded bowel movement. Meanwhile, she was yellow in complexion, thin in figure and exhausted in spirit with redness at tongue tip and white coating from inspection, deep and thready in pulse diagnosis. The patient was initially administrated with Xiaochaihu Decoction for three days, which resulted smoothing in the stomach and growing appetite as she described. Next, Zhang was given the same formula for three days more with no significant effects. When I tried to figure out the reason, I was told that she felt quite annoyed and restless, with tendency to sweat after taking the decoction this time. All of a sudden, I recalled one article from Treatise on Febrile Diseases, which states: For patients with persistent vomiting, discomfort in the stomach, low but slightly annoyed in spirit, one should use Dachaihu Decoction [4]. In spite of persistent vomiting, the rest symptoms all fit in the article. Hence, Dachaihu Decoction was applied and modified as follows: Radix Bupleuri 15 g, Radix Scutellariae 10 g, Rhizoma Pinelliae Praeparata 12 g, Pericarpium Citri Reticulatae 10 g, Radix Paeoniae Alba 15 g, Radix et Rhizoma Rhei Praeparata 9 g, Radix Codonopsis 12 g, Rhizoma Zingiberis Recens 15 g, Radix et Rhizoma Glycyrrhizae 6 g, Fructus Setariae Germinatus 10 g, Fructus Hordei Germinatus $10 \mathrm{~g}$, and Endothelium Corneum Gigeriae Galli $10 \mathrm{~g}$. In the formula, Rhizoma Zingiberis Recens was applied in large dosage not for the vomiting as usual but for her low in spirit by helping Radix Bupleuri and Radix Scutellariae to clear away stagnated heat in Shaoyang with its spicy and warm nature, Rhizoma Pinelliae Praeparata for harmonizing stomach, relieving oppression and preventing vomiting, Radix Paeoniae Alba for relaxing spasm and releving pain, and Radix et Rhizoma Rhei Praeparata for removing excess Fu syndrome. Meanwhile, in consideration of her age, Radix et Rhizoma Glycyrrhizae and Radix Codonopsis were applied to invigorate spleen and strengthen the body resistance. All the symptoms were relieved after taking the latest formula for three days. Since her hepatolithiasis was highly indicated, the patient was suggested to maintain light diet with less oily food and regular administration of Yidan Tablet. No occurrence was reported in the one-year follow-up.

Comment: Dachaihu Decoction has significant antibacterial and anti-inflammatory, cholagogue and calculus removing effects [5], which is frequently used in the treatment of cholecystitis and cholelithiasis in clinic. The case mentioned above is not very typical on account of its imperfection in Western diagnosis and atypical symptoms. Thus, it is suggested that the clinical application of Dachaihu Decoction should not be confined to Western diagnosis, and more emphasis should be given to the comprehension of articles from Treatise on Febrile Diseases, for instance: in this case, discomfort in the stomach, low but slightly annoyed in spirit, scanty but inhibited diarrhea all indicate obstruction of Fu qi in Yangming.

\section{REFERENCES}

[1] Z. J. Zhang, "Theory of Synopsis of Golden Chamber," Publishing House of Chinese Medicine, Beijing, 2006.

[2] Z. J. Zhang, “Treatise on Febrile Diseases," Shanghai People’S Publishing House, Shanghai, 1976.

[3] P. J. Yang, "Spiritual Axis,” Xueyuan Publishing House, Beijing, 2008. 
[4] Z. J. Zhang, “Treatise on Febrile Diseases," Shanghai People's Publishing House, Shanghai, 1976.

[5] W. L. Deng, "Pharmacology of TCM Formula and Its
Application,” Chongqing Publishing House, Chongqing, 1990. 